\title{
Systemic Embolism in Mitral Stenosis : Relation to the Size of the Left Atrial Appendix
}

\author{
WALTER SOMERVILLE,* M.D., F.R.C.P. ; R. JANE CHAMBERS, † M.B., B.S.
}

Brit. med. F., 1964, 2, 1167-1169

A suspicion has existed for many years that systemic embolism is linked with a large left atrial appendix in patients with rheumatic mitral valve disease (Madden, 1949). For this reason Bailey et al. (1952) advised that it be amputated at mitral valvotomy. A few years later Belcher and Somerville (1955) added their support for amputation as a protection against future embolism. Surgical opinion remains divided on this technical point, one reason being that no convincing evidence has been produced incriminating the left atrial appendix.

The object of this investigation was to find out whether any relation exists between systemic embolism and the size of the left atrial appendix in patients with mitral stenosis. The problem was investigated by noting ( $a$ ) the incidence of systemic embolism in mitral stenosis when the left atrial appendix was small, medium, and large respectively, and $(b)$ the size of the left atrial appendix in patients who had developed systemic embolism. These observations were made over a period of one

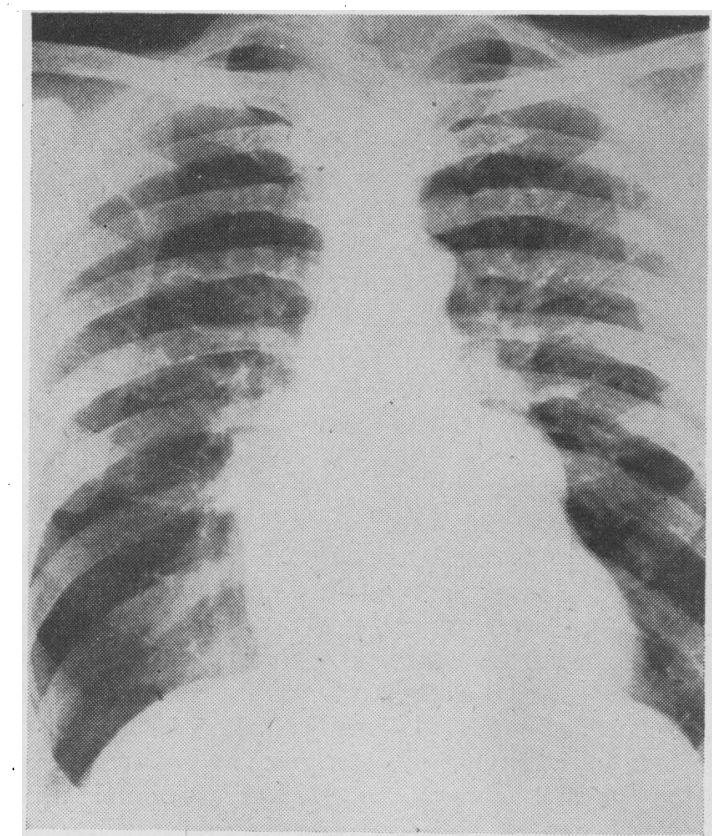

Frg. 1.-Radiograph showing prominent bulge on the left cardiac border caused by a large left atrial appendix.

to seven years in 525 patients with mitral stenosis who had a transventricular valvotomy performed. All were under the care of and were examined by one or both of us.

The diagnosis of systemic embolism was based on the customary clinical features which either were observed by us or were an inherent part of the clinical history. The size of the left atrial appendix was graded as small (or absent), medium, or large, according to its appearance at operation. The operations were performed by a number of surgeons at the Middlesex or Harefield Hospital. These surgeons had already

* Physician, Cardiac Department, the Middlesex Hospital, London. + Medical Registrar, Harefield Hospital, Harefield, Middlesex. performed between them 385 mitral valvotomies by the transatrial route by the time the present series began and so were familiar with the appearance of the left atrial appendix. This structure presents in a variety of different shapes, but there was usually no difficulty in classifying any particular appendix into one of the three categories of small, medium, and large. An independent assessment had been made before operation, based on the appearance of the left atrial curves on the posteroanterior chest radiograph. When this was prominent (Fig. 1) it was a reliable indicator of a large appendix. A concavity instead of a convexity usually meant a small appendix or the site of an appendix amputated at a previous valvotomy. The radiological assessment has fallacies: the bulge of a large appendix can be masked by cardiac enlargement, rotation, or displacement ; a long serpentine appendix may not project on the left border of the heart; the curve of a large main pulmonary artery and its left descending branch may incorporate the appendix bulge.

\section{Results}

The incidence of systemic embolism in mitral stenosis when the size of the left atrial appendix was small, medium, and large respectively is shown in Fig. 2. The figures indicate that emboli were more than three times as common when the appendix was large than when it was small. The differences a $\mathbf{r}$ e highly significant $(\mathrm{P}<0.001)$.

The size of the left atrial appendix in patients with and without a history of embolism respectively is illustrated in Fig. 3, which shows that the appendix was larger in

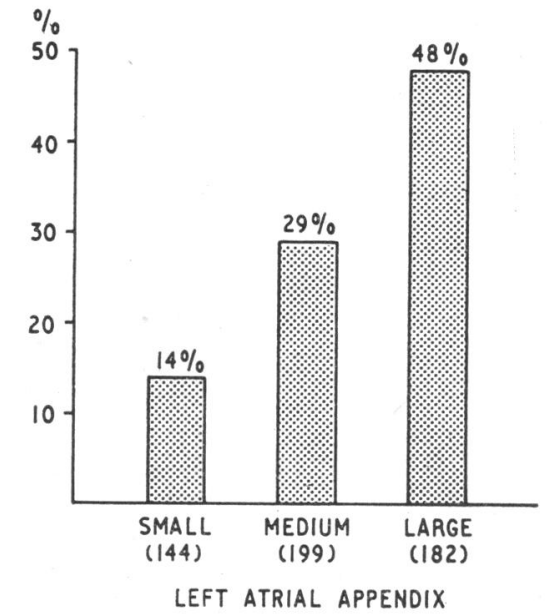

FIG. 2.- Incidence of embolism in relation to size of left atrial appendix in 525 cases of mitral stenosis. the embolic group.

The preponderance of larger appendices in the embolic group was further accentuated when those patients who sustained two or more emboli were considered separately.

\section{Discussion}

The results of this investigation confirm that an intimate relation exists between systemic embolism and the size of the left atrial appendix in mitral stenosis-the larger the appendix the more likely an embolism. However, a small or absent left atrial appendix in no sense conveys immunity against embolism, while a person with a large appendix may run his course without 


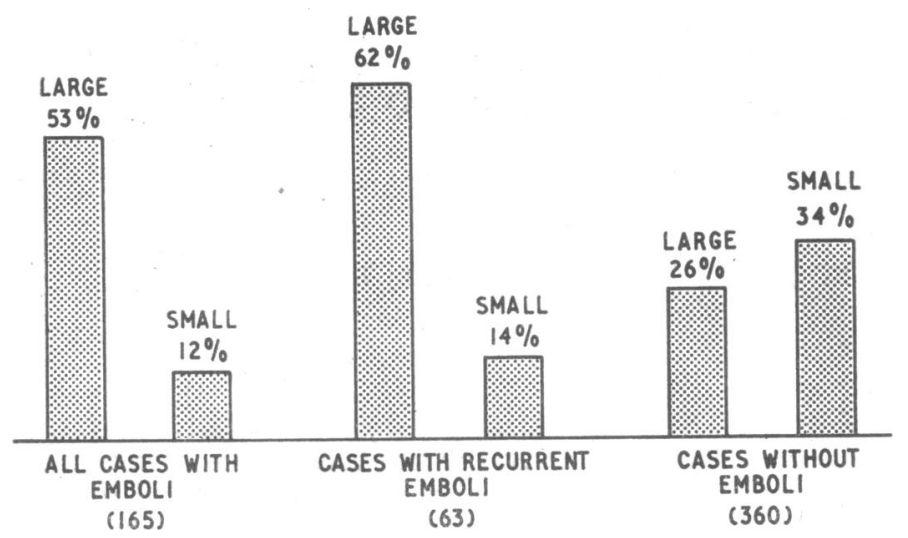

FIG. 3.-Size of left atrial appendix in patients with and without emboli.

this complication. Other factors are clearly involved in embolus-formation, particularly rhythm changes, and it is not our present purpose to consider all of them. The longer a person with mitral stenosis survives the greater the chance of developing an embolus, and this likelihood depends to a great extent on the size of the left atrial appendix. The age distribution of 182 patients with mitral stenosis and a large appendix is set out in Fig. 4, and of 144 patients with a small appendix

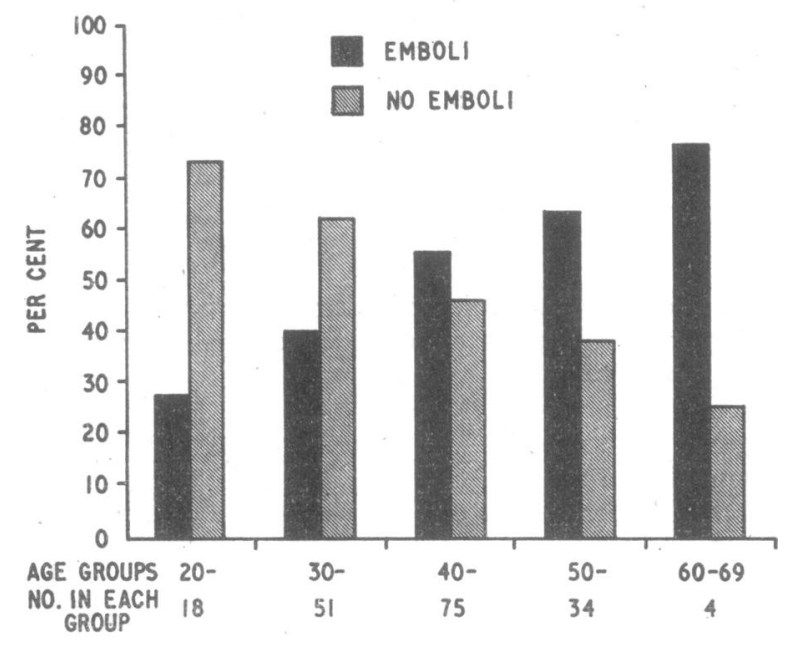

FIG. 4.-Age distribution of 182 patients with mitral stenosis and a large left atrial appendix.

in Fig. 5. The incidence of paroxysmal and permanent atrial fibrillation, cardiac enlargement, and a history of congestive heart failure was similar in both groups. The occurrence of embolism is seen to rise steeply in successive decades when the appendix is large $(P<0.001)$, but not when it is small $(0.10>\mathrm{P}>0.02)$.

The reasons underlying the relation between embolism and a large left atrial appendix are not altogether clear. The tendency for thrombi to form in the appendix is the most important one. The appendix is a cul-de-sac with rough walls, its cavity sometimes criss-crossed by cords and strands. It contracts with the atria in sinus rhythm, but when the atria fibrillate it loses most if not all of its contractility. Sluggish blood-flow must then predispose to thrombus formation. Indeed, it is difficult to understand why thrombi do not invariably form in the large appendix, particularly in the long narrow sinuous type. A systemic embolism in mitral stenosis invariably originates as a thrombus, usually in the left atrium or its appendix, the rare exceptions being a left ventricular mural thrombus and a paradoxical embolism via an atrial septal defect. This fact is not contradicted by the low incidence $(16 \%)$ of thrombus found in the left atrium and its appendix at mitral valvotomy (Belcher and Somerville, 1955) or at necropsy (58\%) (Graef et al., 1937), for there is incontrovertible evidence that a thrombus may be expelled from the left atrium or its appendix without leaving a trace.

It is not clear why the left atrial appendix enlarges disproportionately in certain cases of mitral-valve disease. It is not related to the size of the left atrium or the severity of the mitral stenosis. Rheumatic infection intensified in the appendicular wall may weaken it and allow distension disproportional to the atrial enlargement, but histological proof of excessive

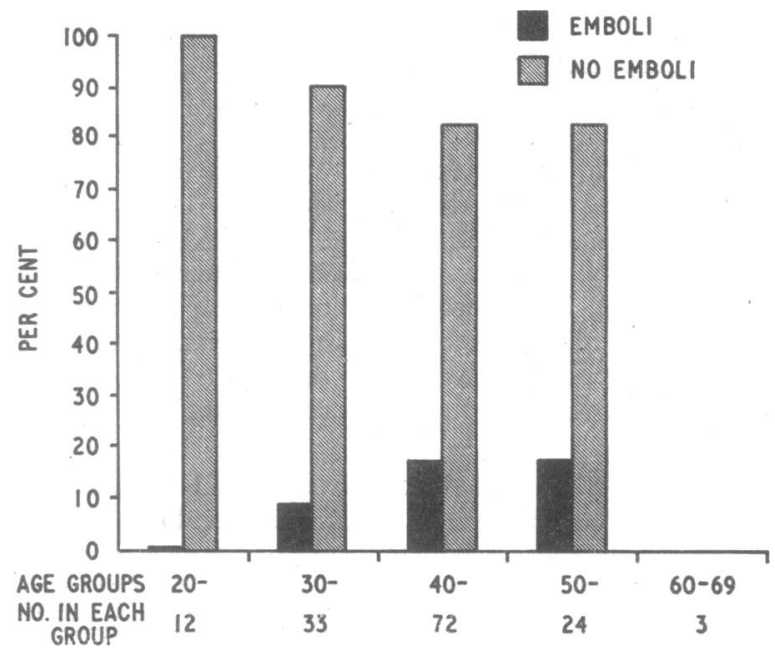

FIg. 5.-Age distribution of 144 patients with mitral stenosis and a small left atrial appendix.

rheumatic activity in the appendix was not shown in this series. Neither was there any difference between the incidence of Aschoff bodies in the appendix of embolic and non-embolic cases.

Should the left atrial appendix be amputated at mitral valvotomy? Personal interrogation of many cardiac surgeons disclosed different answers. The main reason given for not amputating was to preserve a convenient entrance to the mitral valve in the event of a second valvotomy. There is strong evidence that a good valvotomy protects against systemic embolism, but whether this protection is maximal deserves further inquiry. A survey of 910 mitral valvotomies, including the present 525 transventricular and 385 transatrial operations performed on patients in our care, revealed 15 who developed systemic embolism months or years after operation. At the second operation the appendix was still present in 11 ; in eight of these the appendix was large. Bannister (1960) has marshalled strong arguments against postponing valvotomy in patients with moderate mitral stenosis and trivial symptoms, showing in a careful retrospective study that deferring operation leaves them exposed to a high risk of systemic embolism. He suggested that excision of a large left atrial appendix may be as important as relieving the stenosis. We now believe that amputation of the appendix conveys additional protection against embolism and should form part of the operation of mitral valvotomy.

Confirmatory evidence that a large left atrial appendix is a hazard even when the mitral valve is normal is provided by the frequency of systemic embolism in this uncommon congenital anomaly (Parmley, 1962 ; Williams, 1963). Widening experience with rheumatic mitral-valve disease strongly suggests that a number of patients presenting with systemic emboli have only trivial valve involvement, slight stenosis or incompetence or both, but the left atrial appendix is disproportionately large. The evidence is now convincing that further emboli are likely to occur if operation is withheld on the grounds that the valve involvement is not severe enough to justify it. It has been our practice to press for surgical treatment of those patients with a large appendix in preference to long-term anticoagulation even though the latter is known to 
convey good protection against systemic emboli (Szekely, 1964). The operation designed to prevent further emboli consists of amputation of the appendix and correction when possible of the valve disease, even though it is trivial. Left atrial appendicectomy was recommended by Madden (1949), but he reported on only two cases, the results were not convincing, and he did not press home his argument.

\section{Summary}

Systemic embolism in patients with mitral stenosis was more than three times commoner when the left atrial appendix was large than when small.

Conversely, the left atrial appendix was larger in patients with a history of embolism than in those without it.

The occurrence of embolism rose steeply in successive decades when the appendix was large but not when it was small.

The left atrial appendix should be amputated at mitral valvotomy as an extra precaution against embolism.
A number of patients presenting with systemic embolism and only slight rheumatic mitral-valve involvement have disproportionate enlargement of the left atrial appendix. Amputation of the appendix and relief of mitral stenosis, however slight, is recommended here as a protection against further emboli.

We are grateful to the physicians and surgeons of the Middlesex and Harefield Hospitals, to Dr. A. Kardalinos, and to Mr. R. F. J. Withers, of the Department of Biology as Applied to Medicine, Middlesex Hospital Medical School, for advice and assistance.

\section{REFERENCES}

Bailey, C. P., Olsen, A. K., Keown, K. K., Nichols, H. T., and Jamison, W. L. (1952). f. Amer. med. Ass., 149, 1085.

Bannister, R. G. (1960). Lancet, 2, 329.

Belcher, J. R., and Somerville, W. (1955). Brit. med. F., 2, 1000.

Graef, I., Berger, A. R., Bunim, J. J., and de la Chapelle, C. E. (1937). Arch. Path., $24,344$.

Madden, J. L. (1949). Э. Amer. med. Ass., 140, 769.

Parmley, L. F. (1962). Circulation, 25, 553 .

Szekely, P. (1964). Brit. med. ₹., 1, 1209.

Williams, W. G. (1963). Brit. Heart Ұ., 25, 637.

\title{
Mitral Stenosis and Systemic Emboli
}

\author{
DAVID LILLICRAP,* M.A., B.M., M.R.C.P.; ALINA PIESOWICZ, $†$ M.B., M.R.C.P., D.C.H.
}

Systemic emboli in patients with mitral stenosis are a common event. In patients with mitral stenosis of all grades of severity Wood (1954) reported an incidence of $14 \%$. Bannister (1960) found that 22 patients out of 114 had systemic emboli over a period of 10 years. All these patients initially had mitral stenosis that was not severe enough to warrant valvotomy.

Mitral valvotomy is performed to relieve significant mechanical obstruction at the valve. If a patient with mitral stenosis has an embolus this is usually accepted as an additional reason for valvotomy. Mitral valvotomies have also been performed in patients in whom the degree of obstruction in itself was not sufficient to warrant operation, but in whom systemic emboli had occurred. It has also been suggested that in patients over the age of 40 who have moderate mitral stenosis but only trivial symptoms the risk of systemic emboli is high and valvotomy should be performed before any emboli occur (Bannister, 1960). This is reasonable only if the risk at operation is small, there is no detrimental effect of operation on the survivors, and the incidence of post-operative systemic emboli is reduced. With this in mind we have followed up 84 patients who had mitral valvotomies at Guy's Hospital in 1957, 1958, and 1959. All patients were over the age of 40 . The number of patients under 40 was too small for any comparisons and they were therefore excluded. We also excluded patients who had significant lesions of other valves and patients who had had previous mitral valvotomies. Six patients had moderate mitral incompetence and eight had slight incompetence. At operation it was confirmed that mitral stenosis was the dominant lesion.

Clinical assessment of disability was made pre-operatively and at yearly intervals, using the grades as defined by Baker

* Senior Registrar, Guy's Hospital, London.

+ Now Senior Registrar, Hospital for Sick Children, Great Ormond Street. London. et al. (1952). These are : grade 0, no disability; grade 1, slight disability ; grade 2 , moderate disability ; grade 3 , severe disability ; and grade 4, total incapacity. Improvement of two or more grades or post-operative grade 0 has been classed under good operative results; no improvement or improvement by one grade as an unsatisfactory result.

Patients are divided into two groups-those without preoperative emboli and those who had pre-operative embolithe occurrence of emboli in all cases being a clinical diagnosis, based on the abrupt onset of symptoms and signs consistent with the occlusion of an artery with rapid partial or complete recovery. The results are reported and compared. All but four patients were seen personally at the three-year follow-up.

\section{Results of Mitral Valvotomy}

\section{Patients Without Pre-operative Emboli}

Of the 84 patients, 48 were in this group. Three died as a direct result of the operation-one in grade 3 died of multiple emboli 24 hours after operation; one in grade 3 died three days after operation from left-sided heart failure, and the third patient was in grade 4 and died of haemorrhagic tracheobronchitis. Table I shows the results of the follow-up in the remaining 45 patients. Thus besides the three opera-

TABle I.-Status of 45 Patients Without Pre-operative Emboli, at Each

\begin{tabular}{|c|c|c|c|c|c|c|}
\hline \multicolumn{3}{|c|}{ Exercise Tolerance } & \multirow{2}{*}{$\begin{array}{c}\text { Pre-op. } \\
0 \\
1 \\
18 \\
26 \\
0\end{array}$} & \multirow{2}{*}{$\begin{array}{c}\text { 1st Year } \\
15 \\
16 \\
7 \\
3 \\
1\end{array}$} & \multirow{2}{*}{$\begin{array}{c}\text { 2nd Year } \\
15 \\
16 \\
4 \\
5 \\
0\end{array}$} & \multirow{2}{*}{$\begin{array}{c}\text { 3rd Year } \\
15 \\
16 \\
3 \\
3 \\
1\end{array}$} \\
\hline $\begin{array}{cc}\text { Grade } & 0 \\
\Rightarrow & 1 \\
\# & 2 \\
\# & 3 \\
, & 4\end{array}$ & $\begin{array}{l}\cdots \\
\cdots \\
\cdots \\
\cdots\end{array}$ & $\begin{array}{l}\cdots \\
\cdots \\
\cdots\end{array}$ & & & & \\
\hline Deaths & $\ldots$ & $\ldots$ & - & 3 & 5 & 7 \\
\hline
\end{tabular}

